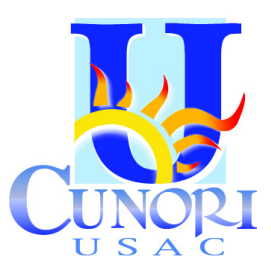

\author{
Revista Ciencia Multidisciplinaria CUNORI \\ http://revistacunori.com \\ DOI: https://doi.org/10.36314/cunori.v2i1.53 \\ ISSN: 2617- 474X (impresa) / 2617- 4758 (en línea)
}

Como citar el artículo

Escalante, A. (2018). El Ejercicio Profesional Supervisado como método de evaluación final en la facultad de ciencias económicas. Revista Ciencia Multidisciplinaria CUNORI, 2(1). 53-59. https://doi.org/10.36314/cunori.v2i1.53

\title{
El Ejercicio Profesional Supervisado como método de evaluación final en la Facultad de Ciencias Económicas
}

\section{The professional exercise as a method of final evaluation in the Faculty of Economic Sciences}

\author{
Ana Lorena Escalante Pérez* \\ Facultad de Ciencias Económicas de la Universidad de San Carlos de Guatemala \\ Recibido: 10 de junio de 2018 Aceptado: 08 de agosto de 2018 \\ Disponible en internet el 17 de agosto de 2018 \\ *Autor para correspondencia. \\ Correo electrónico: aescape1@yahoo.com
}

Resumen

耳 1 artículo analiza el Ejercicio Profesional Supervisado -EPS, para estudiantes de contaduría pública, administración de em- 1 presas y economía, impartidas por la Facultad de Ciencias Económicas de la USAC. Al realizar el análisis, se detectó que influyen elementos generadores de diversos problemas, situación que se ve reflejada en la deserción y repitencia en un promedio del 46\% de estudiantes inscritos en el EPS, según historial de los últimos diez años. (Ejercicio Profesional Supervisado, 2017) Los principales resultados de la investigación es la baja formación docente, cobertura parcial de mobiliario y equipo, limitadas opciones de graduación y desactualización de estudiantes con largos períodos de ausencia universitaria. El método general de investigación utilizado fue el científico, se aplicó el análisis y la síntesis. Se realizó investigación mixta al combinar datos cuantitativos y cualitativos. La primera basada en el post positivismo y la segunda en la teoría fundamentada. (Strauss \& Corbin, 2002) En la investigación se determinó que de los 414 inscritos hay 220 estudiantes que ingresaron al EPS en el primer semestre del año 2017 , con 9 a 41 años de antigüedad dentro de la carrera, la situación reflejó que estos se encontraron desactualizados con relación a los requerimientos del programa. En la encuesta realizada a docentes estos afirmaron que no han podido asistir a las capacitaciones por el factor tiempo. Otra de las debilidades detectadas fueron las limitadas opciones para graduación a las que tiene acceso el estudiante.

Palabras clave: programas de evaluación final, sistema de evaluación, ejercicio profesional supervisado

Abstract

The article analyzes the Supervised Professional Exercise-EPS, for students of public accounting, business administration and

economics, taught by the Faculty of Economic Sciences of the USAC. When carrying out the analysis, it was detected that elements generating various problems influence, situation that is reflected in the dropout and repetition in an average of $46 \%$ of students enrolled in the EPS, according to the last ten years' record. (Supervised Professional Exercise, 2017) The main results of the research are low teacher training, partial coverage of furniture and equipment, limited graduation options and out-of-date students with long periods of university absence. The general method of investigation used was the scientist, the analysis and the synthesis were applied. Mixed research was carried out by combining quantitative and qualitative data. The first based on post positivism and the second based on theory. (Strauss \& Corbin, 2002) In the investigation it was determined that of the 414 enrolled there are 220 students who joined the EPS in the first semester of 2017, with 9 to 41 years of seniority within the career, the situation reflected that these were outdated in relation to the requirements of the program. In the survey made to teachers, they affirmed that they 
have not been able to attend the trainings due to the time factor. Another of the weaknesses detected was the limited options for graduation to which the student has access.

Keywords: final evaluation programs, evaluation system, supervised professional practice

\section{Introducción}

El problema fue planteado al hacer la pregunta ¿Qué implicaciones posee el Ejercicio Profesional Supervisado como método final de evaluación, en la facultad de ciencias económicas de la USAC 2017 ? Para lo cual se utilizaron encuestas dirigidas a estudiantes y docentes, entrevistas a expertos, las entrevistas por saturación a estudiantes que desempeñaron trabajo de campo y a profesionales egresados por el método de EPS, también se realizaron observaciones directas durante el desarrollo de la investigación. Al determinar el número de estudiantes que no logró culminar el proceso de graduación se dejó de cumplir con los objetivos para los que fue creado el programa de EPS, donde el propósito es "dar oportunidad al estudiante de hacer aplicación de los conocimientos adquiridos, por medio de prácticas" (Reglamento del Ejercicio Profesional Supervisado, 1982:6). De la misma manera se está dejando de cumplir con los estudiantes, debido a que no se le ha prestado atención a los que quedaron en rezago.

La fundamentación posee principios teóricos donde se incluyó estudios realizados por el Espacio Europeo de Educación Superior y el proyecto Tunning América Latina, para la evaluación de proyectos finales de carrera en América Latina. Otros estudios de autores nacionales como Mario Rodríguez Acosta, de la misma manera se utilizaron las leyes y reglamentos del programa de la facultad, de la universidad y las leyes nacionales. El objetivo principal de la investigación es evaluar el desarrollo del programa de EPS como método de evaluación final en la facultad de Ciencias Económicas de la Universidad de San Carlos de Guatemala, durante el primer semestre del año 2017.

\section{Material y métodos}

Para (Behair, 2008:34) "el método para la obtención del conocimiento denominado científico es un procedimiento riguroso, de orden lógico, cuyo propósito es demostrar el valor de la verdad de ciertos enunciados". Para el estudio del programa se utilizó el científico como método general y como particulares se utilizó el inductivo para la sección cualitativa, y el hipotético deductivo para la parte cuantitativa. La metodología que se utilizó en la investigación fue la mixta, basada en (Hernández, 2014:532), la que describe un conjunto de procesos de recolección, análisis y vinculación de datos cuantitativos y cualitativos en un mismo estudio o una serie de investigaciones para responder a un planteamiento del problema.

Para (Cascante, 2011:11), los enfoques son formas de llevar a cabo un proceso de acción, desde la visión y los objetivos hasta sus particularidades; estos corresponden a valores, conceptos y tendencias singulares. En el caso de la investigación, a lo largo de la historia se conocen principalmente dos, el cuantitativo y el cualitativo, el mixto surge a partir de la combinación de ambos. Para determinar la muestra se aplicó lo expuesto por (Bernal, 2000:159), explica que "es la parte de la población que se selecciona y de la cual se obtiene la información, para el desarrollo del estudio sobre la cual se efectuaron, la medición y la observación de las variables objeto de estudio". 
La población consistió en 414 estudiantes inscritos en el programa de EPS 2017, denominado seminario general, y 29 profesores. En la investigación se utilizó instrumentos de recolección de informaciones diversas, la boleta de encuesta para estudiantes. Se entrevistó a estudiantes que realizaron trabajo de campo y trabajo de gabinete, se realizaron entrevistas a profesionales egresados por EPS año 2014 y así mismo para los expertos, durante toda la observación se trabajó con guía de observación.

El vaciado de la información recolectada, fue tabulada con el uso de herramientas electrónicas de Excel, seguidamente se realizaron cuadros y gráficas donde se sintetizó la información y se finalizó con el análisis respectivo. Para las medidas representativas de un conjunto de datos se utilizó la moda, mediana y el promedio, estas sirvieron para determinar los valores que más se repiten en la investigación, para después identificar el valor que se encuentra por encima de la media y el valor que divide en dos los datos numéricos.

Se analizó la desviación estándar, la varianza y el rango. Donde la desviación estándar es "el promedio de desviaciones o dispersiones de las puntuaciones respecto a la media" (Bernal Torres, 2000), esta medición permitió determinar la existencia de la relación entre antigüedad del estudiante y la calificación final que obtuvo en el proceso de cada seminario.

Se trabajó con estas medidas los rangos de las calificaciones, para identificar hacia donde se dispersan, y donde se encuentran concentradas, información que fue tomada de los archivos de calificaciones que poseen en el EPS del primer semestre 2017. Se procedió a describir cada objeto y fenómeno observado, al destacar las características esenciales de acuerdo a la percepción de la investigadora, se utilizó un cuaderno para plasmar lo que parecía interesante, los acontecimientos que se logran observar, para luego ser descritos en la redacción de lo investigado.

\section{Resultados}

Existe contradicción con el desarrollo de la carrera y el ejercicio profesional supervisado. El contenido académico en el área común representa el bagaje conceptual que el estudiante al culminar debiera manejar y además son bases fundamentales para el EPS, sin embargo los resultados son contradictorios, derivado a que los estudiantes del programa de EPS no realizan una aprehensión de los conocimientos que se ofrecen en cada curso y menos desempeñar una labor investigativa de carácter autodidáctico. Adicionalmente se verificó que existe incongruencias entre el programa de EPS y área común, siendo esta última precisamente en donde se ubican los cursos que sustentan el área social humanista del programa del EPS. (Departamento de planificación y desarrollo educativo. Fac. de C.E., 2008:21)

Durante la carrera, los temas son relacionados de forma macroeconómica, mientras que el epesista aplica los conocimientos a las microfincas, familiares, y empresas artesanales del área rural de Guatemala, actividades donde no se llevan controles y registros de su producción, entonces se visualiza un oposición entre lo aprendido y lo puesto en práctica por parte del estudiantado y un conflicto institucional debido a que no se cumplen los objetivos para los cuales fue creado el programa de EPS (CSU Reglamento del Ejercicio Profesional Supervisado, 1982:5-8). 
Uno de los grandes problemas detectados fue la saturación de estudiantes por salón de clases, donde se practicó de manera limitada el trabajo en equipo, aunado al docente que mantuvo una actitud pasiva ante los grupos. En el cuadro uno, refleja el número de estudiantes por año y por carrera inscritos en el EPS de C.E. de la Usac.

\begin{tabular}{rrrcr}
\hline Años & Auditoría & Admón. de empresas & Economía & Totales \\
\hline 2007 & 315 & 220 & 7 & 542 \\
2008 & 220 & 131 & 4 & 355 \\
2009 & 291 & 195 & 7 & 493 \\
2010 & 289 & 243 & 9 & 541 \\
2011 & 451 & 297 & 10 & 758 \\
2012 & 545 & 389 & 9 & 943 \\
2013 & 497 & 304 & 21 & 822 \\
2014 & 456 & 389 & 26 & 871 \\
2015 & 474 & 431 & 15 & 920 \\
2016 & 483 & 415 & 13 & 911 \\
\hline
\end{tabular}

Fuente: archivos de EPS año 2016.

Cuadro 1: Guatemala, estudiantes inscritos en el EPS, facultad de C.E. USAC. Años 2007 a 2016.

El cuadro refleja el movimiento de estudiantes que se inscribieron en el programa con el objetivo de obtener un título profesional. Se mantiene un promedio de 482, hasta el año 2011. Al año 2012 se da un incremento a 943 estudiantes debido a que se suman los egresados de plan fin de semana.

Se aprecia la mayor cantidad de auditores y administradores de empresas, debido a que en la iniciativa privada se da la contratación de los egresados de esta profesión, caso diferente para los economistas, debido a que la carrera no tiene demanda en cuanto a oportunidades laborales en esta área. A continuación, se presentan el cuadro dos donde se visualiza la afluencia de estudiantes al inicio de cada año y la comparación con los que finalizan el proceso con la graduación. 


\begin{tabular}{cccc}
\hline Años & $\begin{array}{c}\text { Estudiantes inscritos al inicio del } \\
\text { proceso de EPS }\end{array}$ & $\begin{array}{c}\text { Estudiantes que culminan el } \\
\text { proceso de seminario }\end{array}$ & $\begin{array}{c}\text { Relación estudiantes que culminan el } \\
\text { proceso comparado con los inscritos } \\
\text { en el EPS\% }\end{array}$ \\
\hline 2007 & 542 & 305 & 56 \\
2008 & 355 & 227 & 64 \\
2009 & 493 & 278 & 56 \\
2010 & 541 & 346 & 64 \\
2011 & 758 & 386 & 51 \\
2012 & 943 & 493 & 52 \\
2013 & 822 & 419 & 51 \\
2014 & 871 & 395 & 45 \\
2015 & 920 & 445 & 48 \\
2016 & 911 & 439 & 48 \\
\hline
\end{tabular}

Fuente: datos de archivos de EPS de los años 2007- 2016.

Cuadro 2: Comparación de estudiantes inscritos al inicio del programa de EPS, con los que realizan trabajo de campo. Años 2007 al 2016.

La información refleja una fuerte baja de estudiantes que no lograron culminar los procesos de evaluación en los seminarios, los estudiantes que realizaron trabajo de campo son los que generalmente culminan con su graduación. Lo expuesto en los párrafos anteriores se contradice con los objetivos de la Facultad, de la Universidad y el programa de EPS, debido a que no se llega en su totalidad a proporcionar al estudiante la facilidad para que pueda realizar investigación y al mismo tiempo pueda interactuar y contribuir con las comunidades vulnerables y desposeídas del país, no así en la contribución de resolver la problemática Nacional (CSU, Leyes y reglamentos de la Usac, 2006:10).

Con respecto a los resultados de la encuesta a docentes del programa de EPS, las respuestas evidencian que el docente no recibe capacitaciones que le permitan desempeñarse efectivamente, pero no es porque no existan capacitaciones si no que no asiste por falta de tiempo, debido a que en el programa no se interrumpen actividades, además el docente de EPS, expresó sentirse desmotivado debido a que en la facultad no posee una programación de horarios abiertos para las capacitaciones.

Se realizó entrevista a personal del Departamento de Planificación y Desarrollo Educativo, donde se indicó que la facultad no posee una planificación de capacitación para el personal docente, esta unidad únicamente trasmite las convocatorias de la Dirección de Desarrollo Académico de la Universidad por medio del Sistema de Formación del Profesor Universitario, a través de los correos electrónicos del personal registrado.

Se le preguntó al docente si recibe al inicio del programa el perfil de sus estudiantes, 23 contestaron que no les proporcionan esta información de forma oportuna, debido a que la ficha de ingreso contiene datos elementales del estudiante como los datos personales, padecimiento de enfermedades, datos de estudios, lugar de trabajo y capacitaciones recibidas. Entonces es necesario que el docente conozca con anticipación a quienes atenderá en los seminarios de inducción, pues desconoce si el estudiante posee una capacidad diferente, o padece alguna enfermedad, se ve la necesidad de proporcionar este tipo de Revista del Centro Universitario de Oriente CUNORI-USAC-GUATEMALA 
información para que el docente en caso de emergencia esté enterado y sepa a quien acudir, si el estudiante necesita una atención especial para que pueda desarrollarse con los demás estudiantes y que al mismo tiempo el programa de EPS sea incluyente.

El espacio en las aulas es limitado no permite que el estudiante y docente interactúen para el desarrollo de las actividades del programa, siendo este el más perjudicado, situación que se ve reflejada en la deserción y repitencia. Se agrega que en la actualidad el estudiante no posee acceso a internet dentro de las instalaciones donde se imparte el programa de EPS, así como el total de docentes (29) únicamente 13 poseen acceso a esta herramienta, lo que se considera una limitante para la interacción virtual con el estudiantado.

\section{Discusión}

Los profesores del programa de EPS, se encuentran desactualizados con relación a los requerimientos de la educación en el presente siglo, la facultad no posee un programa con horario abierto para las capacitaciones, actualmente el docente se encuentra formado académicamente como contador público y auditor, administrador de empresas y economista, preparado para desempeñarse en las empresas, no así como docente, le hace falta conocimientos pedagógicos para interactuar con el estudiante, poseer herramientas para despertar el interés, ser fuente de motivación y encontrar las debilidades del programa para proponer mejoras al sistema, todas encaminadas al beneficio del estudiante.

Los estudiantes que ingresaron al programa de EPS, se encuentran comprendidos entre las edades de 23 años los más jóvenes y 77 años la persona de mayor edad. La comparación de fechas desde que este ingresó a la universidad comparada con el año 2017, fecha de preparación de EPS. Hay estudiantes que poseen de nueve hasta 41 años en las carreras que la facultad imparte, dentro de este rango se encuentran 220 estudiantes, al representar un $72 \%$ de los inscritos. Las personas que en menos tiempo han culminado la carrera están comprendidas entre ocho a seis años, representando la moda en ocho años de carrera.

El grupo con mayor antigüedad se ha ausentado de la facultad por tiempos relativamente largos, los que se encuentran desactualizados, estos crean un alto porcentaje de deserción y repitencia en el programa, a la fecha la facultad no posee un programa de nivelación ante este problema. Según (Chávez 2001:3) el papel de los docentes sería activo y de apoyo hacia el estudiante el cual diseñará estrategias interactivas que promuevan las zonas de desarrollo próximo entre docente y alumno con apoyo estratégico para que el estudiante responda críticamente, que estos procesos, sean más humanos donde se dé el dialogó y la participación.

\section{Referencias bibliográficas}

Behair D. (2008). Introducción a la metodología de la investigación. Universidad Cabo Verde UNICV. Editorial Shalom.

Bernal C. (2000). Metodología de la Investigación para Administración y Economía. Colombia, Santa Fé de Bogotá, Colombia: Nomos S.A. 
Cascante J. (2011). Métodos mixtos de investigación. Guía de estudio. Universidad Estatal a Distancia, Institución Benemérita de la Educación y Cultura.

Chavez A. (2001). Implicaciones educativas de la teoría socio cultural de Vigotski. Revistas Educación. Universidad de Costa Rica, 25 (2), 59 65. https://doi.org/10.15517/revedu.v25i2.3581

CSU Reglamento del Ejercicio Profesional Supervisado. (1982). Reglamento del Ejercicio Profesional Supervisado. Guatemala: Universitaria. CSU, Leyes y reglamentos de la Usac. (2006). Ley orgánica de la Usac. Guatemala.

Departamento de planificación y desarrollo educativo. Fac. de C.E. (2008). Catálogo de estudios 2008 Facultad de Ciencias Económicas. Guatemala: Universitaria.

Ejercicio Profesional Supervisado. (2017). Historial de Archivos de EPS. Universidad de San Carlos de Guatemala.

Hernández, R., Fernández Collado, C., \& Baptista Lucio, P., (2014). Metodología de la investigación (6a. ed.). México: McGraw Hill Education.

Rodríguez M. (2017). Los conflictos en la educación superior en torno al cambio curricular. 1-32. https:// doi.org/10.19137/qs.v21i3.2112

Strauss, A., \& Corbin, J. (2002). Base de la investigación cualitativa. Técnica y procedimientos para desarrollar la teoría fundamentada. Medellín: Sag. Publications Inc.

\section{Sobre la autora}

\section{Ana Lorena Escalante Pérez}

Profesora en la Facultad de Ciencias Económicas de la Universidad de San Carlos de Guatemala, Contadora pública y auditora, con Maestría en economía ambiental y de los recursos naturales y candidata a doctora por la facultad de humanidades de la Universidad de San Carlos de Guatemala.

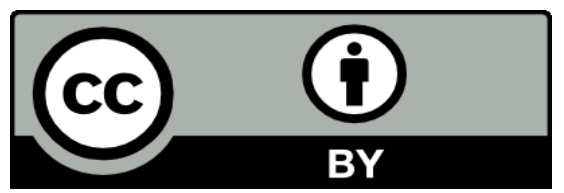

Este texto está protegido por una licencia CreativeCommons 4.0.

Usted es libre para compartir, copiar y redistribuir el material en cualquier medio o formato y adaptar el documento, remezclar, transformar y crear a partir del material para cualquier propósito, incluso comercialmente, siempre que cumpla la condición de atribución: usted debe reconocer el crédito de una obra de manera adecuada, proporcionar un enlace a la licencia, e indicar si se han realizado cambios. Puede hacerlo en cualquier forma razonable, pero no de forma tal que sugiera que tiene el apoyo del licenciante o lo recibe por el uso que hace. 